\title{
39. VISCOUS REMANENT MAGNETIZATION IN BASALT SAMPLES ${ }^{1}$
}

\author{
W. Lowrie ${ }^{2}$ and D.V. Kent, Lamont-Doherty Geological Observatory of Columbia University, Palisades, \\ New York
}

\begin{abstract}
Remanent magnetization measurements were made on four specimens of fresh, fine-to coarse-grained basalt from Site 321 and seven specimens of fresh medium-grained diabase at Hole 319A. The natural remanent magnetizations of these rocks were very unstable, characterized by median destructive fields of less than 100 oe in every sample and large directional changes during partial demagnetization. The samples were able to acquire large viscous remanences (VRM) in the laboratory in a 1.0-oe field. Moreover, the intensity of VRM acquired in the presence of the NRM was twice that acquired under similar conditions but after AF demagnetization. Whether acquired from the demagnetized or NRM state, the VRM acquired in $500 \mathrm{hr}$ amounted to a very large fraction of the NRM intensity, particularly at Hole 319A. These results suggest that the large vertical component of magnetization observed in samples from this site may be in part a VRM acquired while drilling the hole in the presence of the drill pipe ambient field.
\end{abstract}

\section{INTRODUCTION}

Partially oriented specimens, in the form of 1 in. right circular cylinders, were obtained from Core 3 of Hole 319A and Core 14 of Site 321. The specimens were all fresh, and consisted of fine- to very coarse-grained basalt at Hole 321, and medium-grained diabase at Hole 319A (Table 1). The seven specimens from Hole 319A represented a single 15.6-meter thick cooling unit; likewise, at Site 321 the four specimens came from a single homogeneous unit, increasing progressively in grain size with increasing depth (this volume, Chapter 5).

As part of the cooperative investigation of many rock magnetic properties of the Leg 34 basalts, this study was designed to measure the tendency to acquire viscous remanent magnetization (VRM) at Hole 319A and Site 321 and to evaluate its significance with respect to the remanent magnetic properties of the basalts from these sites. Four stages of investigation are involved: (1) measurements of natural remanent magnetic properties, (2) acquisition of VRM in the specimens while they yet carried their original remanences, (3) alternating field (AF) demagnetization of the remanence after $500 \mathrm{hr}$ of VRM acquisition, and (4) acquisition of VRM in the same specimens under the same conditions and after AF demagnetization.

\section{MAGNETIC PROPERTIES}

\section{Natural Remanent Magnetizations}

The intensity of natural remanent magnetization (NRM) was measured in each specimen using a spinner

'Institut für Geophysik, ETH-Zürich, Contribution No. 121.

'Institut für Geophysik, ETH-Hönggerberg, CH-8049 Zürich, Switzerland. magnetometer. The specimens' initial susceptibilities were measured with an AC bridge which was calibrated within 5\% accuracy. Plots of these measured values at each site are shown in Figure 1.

The remanent intensities were similar to those measured onboard ship by Ade-Hall (this volume) and were within the range of NRM intensities previously measured in other DSDP basalts (Lowrie et al., 1973; Lowrie, 1974). The stability of NRM was much lower than usually measured in oceanic basalts (see section on VRM experiments).

The ratio of NRM to susceptibility defines $Q_{n}{ }^{\prime}$, the modified Königsberer ratio. $Q_{n}{ }^{\prime}$ values in this study were found to be small; at Site 321 they ranged from 1.3 to 3.6 , and at Hole $319 \mathrm{~A}$, with one exception, they were all less than 1. These give Königsberger ratios about two orders of magnitude smaller than Ade-Hall reports from shipboard measurements. Since the NRM measurements are in agreement, this discrepancy must be due to differences in susceptibility measurements. The susceptibilities measured in the laboratory are typical of other DSDP samples (Lowrie, 1974), whereas the shipboard values are about two orders of magnitude too low. As no details of the shipboard measurements technique are available, it is impossible at this time to reconcile the enormous discrepancy, but the shipboard measured values (inferred from the NRM and $Q_{n}{ }^{\prime}$ values) are atypically low for oceanic basalts.

\section{Magnetic Mineralogy}

The magnetic mineralogy was examined only cursorily, as a more detailed study is to be reported by others. Thermomagnetic analysis was performed by heating small chips from each specimen in the presence of a strong magnetic field (2-4 koe) in a Curie balance of the vertical motion type. Zero-field observations were made regularly to compensate for weight loss of the specimen. 


\begin{tabular}{|c|c|c|c|c|c|c|c|}
\hline \multirow{2}{*}{$\begin{array}{c}\text { Sample } \\
\text { (Interval in } \mathrm{cm} \text { ) }\end{array}$} & \multicolumn{2}{|c|}{ NRM } & \multicolumn{2}{|c|}{$100 \mathrm{oe}$} & \multirow{2}{*}{\multicolumn{2}{|c|}{ 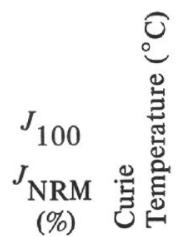 }} & \multirow[b]{2}{*}{ Specimen Description ${ }^{b}$} \\
\hline & $\mathrm{D}^{\mathrm{a}}$ & I & D & I & & & \\
\hline
\end{tabular}

\section{Hole 319A}

$$
3-1,89
$$

$3-2,9$

$3-2,70$

$3-3,64$

$3-4,37$

$3-4,136$

$3-5,72$

Site 321

14-1, 55

$14-2,50$

14-3, 1

$14-4,12$

$\begin{array}{rrrrrr}169 & -38 & 233 & +38 & 17 & 145 \\ 247 & -71 & 310 & +8 & 7 & 130 \\ 166 & -21 & 152 & +48 & 5 & 250 \\ 34 & -72 & 60 & +59 & 13 & 165 \\ 168 & 9 & 216 & -80 & 18 & 145 \\ 248 & 65 & 92 & +79 & 2 & 145 \\ 264 & 26 & 258 & +36 & 24 & 160\end{array}$

145

130

165

145

160
Medium-grained diabase, fresh Medium-grained diabase, fresh Medium-grained diabase, fresh Medium-grained diabase, fresh Medium-grained diabase, fresh Medium-grained diabase, fresh Fine to medium diabase, fresh

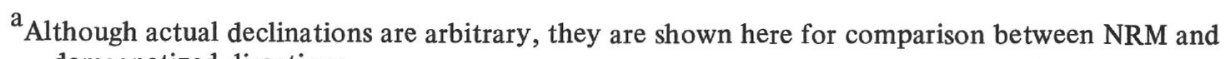
demagnetized directions.

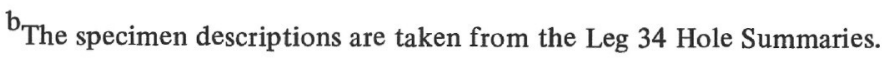

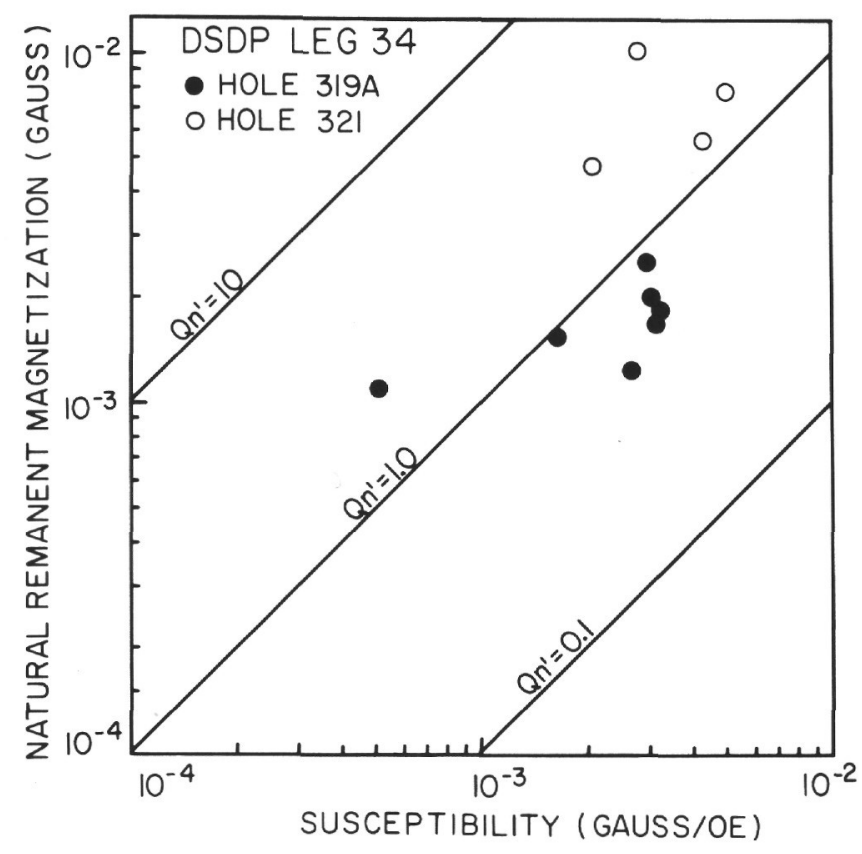

Figure 1. Intensities of NRM and the initial bulk susceptibilities of seven igneous rock samples from Hole $319 \mathrm{~A}$ and four igneous rock samples from Site 321. Diagonal lines show constant values of the ratio of NRM to susceptibility (modified Konigsberger ratio, $\mathrm{Q}_{\mathrm{n}} 1$ ).

A typical thermomagnetic curve showing the variation of strong field magnetization as a function of temperature is shown in Figure 2. Temperature calibration was accurate to within $5^{\circ} \mathrm{C}$; magnetization measurements were uncalibrated.

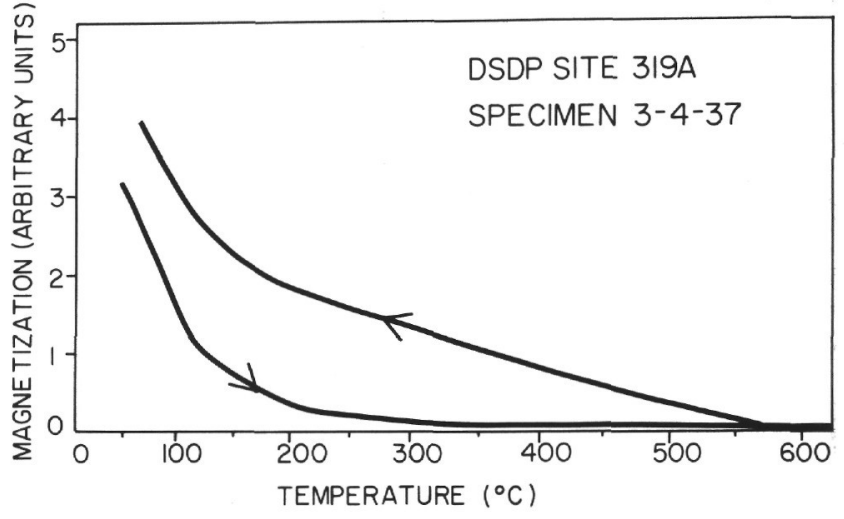

Figure 2. High-field (3 koe) thermomagnetic curve of several chips taken from an igneous rock sample from Hole 319 A. Heating and cooling was performed in air.

The thermomagnetic curves were typical of oceanic basalts of deuteric oxidation class I (Wilson and Watkins, 1967). The Curie temperatures of the initial magnetic mineral in the basalt were generally between $125^{\circ} \mathrm{C}$ and $165^{\circ} \mathrm{C}$, with one value of $250^{\circ} \mathrm{C}$ (Table 1). These are appropriate for an initial magnetic mineralogy dominated by titanomagnetite or titanomaghemite.

On cooling from above $600^{\circ} \mathrm{C}$, two Curie points were evident in most curves. The high temperature value around $550^{\circ} \mathrm{C}$ corresponds to magnetite produced in the exsolution process. The lower Curie point was very close in value to that of the initial magnetic mineral, indicating that the exsolution process was incomplete.

The distinction between titanomagnetite and its cation deficient form, titanomaghemite, is possible from 
thermomagnetic curves performed in a vacuum (Ozima and Ozima, 1971). The present curves were obtained in air, but they strongly indicate titanomagnetite rather than titanomaghemite. In the first place the range of initial Curie temperatures is lower than commonly encountered in titanomaghemites, whose Curie points are raised by the maghemitization process (Ade-Hall et al., 1971). Secondly, the activation energy for exsolution in titanomaghemites $(0.033 \mathrm{ev}$, Ozima and Ozima, 1972) is much lower than in titanomagnetites $(0.5 \mathrm{ev}$, Creer et al., 1970), so that incomplete exsolution is more likely in the latter than in the cation deficient form.

The thermomagnetic data are consistent with the fresh condition of the basalts and showed no systematic variation in Curie point with depth in either hole, although only a very restricted range of sample depths was studied.

\section{VISCOUS REMANENT MAGNETIZATION EXPERIMENTS}

The specimens were placed in a 1-oe field directed along the cylindrical axis ( $z$-axis) of each specimen. Large Helmholtz coils were used to provide a uniform field throughout the region used for this experiment and to cancel other field components. Each specimen was removed from the field-controlled region at periodic intervals for a few minutes, and its remanence was remeasured on the spinner magnetometer. The experiment was continued for $500 \mathrm{hr}$ (3 wks) during which the growth of VRM in each specimen was frequently monitored. The VRM was calculated by subtracting the original NRM measurements from the remanence at each successive stage of VRM acquisition. This first VRM, acquired in the presence of the NRM of the specimen, is referred to as VRM1.

The specimens were next AF demagnetized to remove both the VRM1 and a large portion of the original NRM. The VRM experiments were repeated on the AFdemagnetized specimens under identical conditions to the earlier acquisition. In this case the VRM was calculated at each stage by removing the residual remanence after AF demagnetization. This second VRM, acquired from the AF-demagnetized state, is referred to as VRM2.

\section{NRM Direction and Stability}

The AF-demagnetization characteristics of the specimens were uniformly alike. The VRM1 was removed first, then the original NRM was demagnetized; at fields of 300 oe and higher indications of remagnetization by acquisition of anhysteretic remanence became apparent. This effect was most noticeable at Hole 319A which has extremely unstable magnetization. However, at 100 oe, the remanences were most stable.

The ratio of the remanent intensity after 100-oe AF treatment to the original NRM intensity was very low at Hole $319 \mathrm{~A}$, ranging from $2 \%$ to $24 \%$ and averaging only $12 \%$ (Table 1). Clearly, the NRM contained a very large low-coercivity component that could be influenced by relatively low magnetic fields. The inclinations after 100 oe, although more consistent than the NRM inclinations, were very variable. A negative inclination in
Sample 319A-3-4, $37 \mathrm{~cm}$ is more likely due to accidental specimen inversion than to a field reversal. The inclinations were otherwise all positive, ranging from $8^{\circ}$ to $80^{\circ}$ and averaging $+50^{\circ}$. This range is wider than that found by Ade-Hall (this volume), who found a variation from $42^{\circ}$ to $68^{\circ}$, but the mean value is similar to his $\left(+57^{\circ}\right)$, and dramatically different from that of the present field $\left(-12^{\circ}\right)$ or the axial dipole field, ADF $\left(-25^{\circ}\right)$ at the site.

The ratio of the intensity after 100 oe to the NRM intensity was higher at Site 321 than at Hole 319A (Table 1), but was still low. The ratios ranged from $15 \%$ to $48 \%$ and averaged $29 \%$. At this site also the low-coercivity component of NRM predominated. After 100-oe AF demagnetization, the inclinations averaged $-24^{\circ}$ and were much less dispersed than at Hole 319A. The mean value is close to that measured on shipboard by AdeHall $\left(-21^{\circ}\right)$ and to that of the ADF inclination at the site $\left(-23^{\circ}\right)$.

Close agreement is usually not found between observed remanent inclinations of DSDP basalts and those predicted taking into account plate reconstructions (Lowrie, 1974). In some instances good agreement is found (Lowrie and Hayes, 1975; Larson and Lowrie, in press), but generally discrepancies of $10^{\circ}$ to $20^{\circ}$ are common. The differences between the Site 321 observed and $\mathrm{ADF}$ inclinations (uncorrected for plate motion) are small and may indicate that the basalt was magnetized close to its present latitude.

The presence of large soft components in the NRM at each site, and the low $Q_{\mathrm{n}}{ }^{\prime}$ ratios, have previously been reported in DSDP basalts, many of which could acquire VRM amounting to a significantly large fraction of NRM in only a few weeks (Lowrie, 1973, 1974). The same strongly viscous characteristics were found in each of the Hole 319A and Site 321 specimens.

\section{VRM Characteristics}

The VRM acquisition in all Hole 319A and Site 321 specimens followed the same behavior as shown for a representative specimen in Figure 3. The change of magnetization along the specimen $z$-axis, along which the 1.0-oe field was directed, is depicted as VRM1 (z) in Figure 3. This magnetization increased almost linearly with $\log t$ throughout the period of observation, according to the relationship

$$
\operatorname{VRM} 1(z, t)=\operatorname{VRM} 1(z, 1)+s_{z} \log t
$$

where $s_{\mathbf{z}}$ is a constant, the magnetic viscosity coefficient, and $t$ is the acquisition time in hours. In this same period of time there was a pronounced change in either (or both) the $x$ and $y$ components of magnetization, that is, in the plane perpendicular to the applied field. The same linear relationship with $\log t$ was observed, defining another magnetic viscocity coefficient, $s_{x}$, which was always less than $s_{z}$. As no field component acted in this plane, the change in intensity reflects either relaxation of an existing VRM component in that plane, or reduction of that component of magnetization as very lowcoercivity components remagnetize in the $z$ direction. However, the VRM acquired in the $z$ direction is greater than the accompanying change in the $x-y$ plane. 


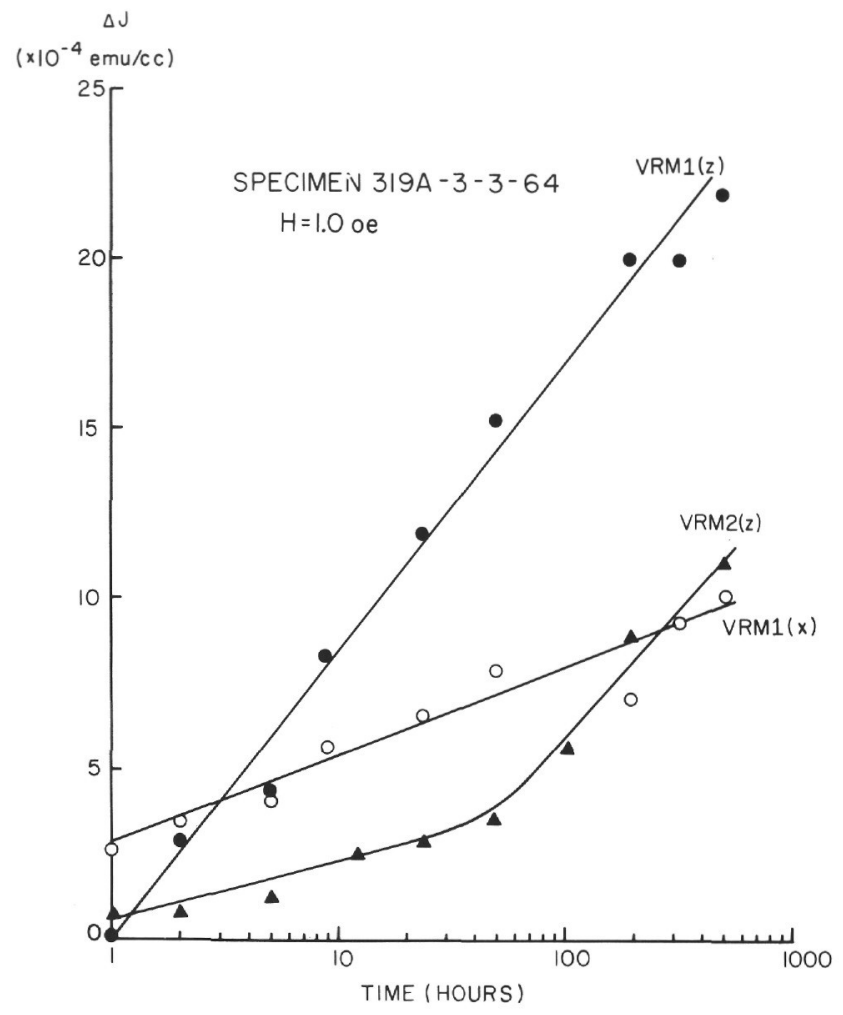

Figure 3. Acquisition of VRM over $500 \mathrm{hr}$ before (VRM1) and after (VRM2) AF demagnetization of NRM in an igneous rock sample from Hole $319 \mathrm{~A}$. A constant magnetic field of 1.0 oe was directed along the specimen $\mathrm{z}$ axis in each experiment although a time-dependent change in remanence was observed also in the perpendidular plane for VRM1 (shown as VRM1 [x]).

After AF demagnetization of the combination of NRM and VRM1, the acquisition of a new VRM (VRM2) follows a different pattern. In this case no systematic variation occurred in the $x$ or $y$ components; as only the $z$ component changed, this remanence is shown as VRM2 $(z)$ in Figure 3. At first VRM2 $(z)$ increased only very slowly with $\log t$. After approximately $50 \mathrm{hr}$, the VRM was acquired more rapidly. In this case

$$
\operatorname{VRM} 2(z, t)=\operatorname{VRM} 2(z, 1)+s_{\mathbf{z}}(t) \cdot \log t
$$

where $s_{z}(t)$, the magnetic viscosity coefficient, is not in this case a constant. It is evident from Figure 3, however, that in each stage (before and after $50 \mathrm{hr}$ ), the acquisition proceeds approximately linearly with $\log t$, so that in each stage a constant value of $s_{\mathbf{z}}$ can be assumed and measured from the slope of the line. The magnetic viscosity coefficient in the early stage $(t<50)$ of acquisition of VRM2 was approximately one-third of the magnetic viscosity coefficient in the later stage $(t>50)$, which in turn was approximately equal to the magnetic viscosity coefficient for the acquisition of VRM1 (z), prior to AF demagnetization (Figure 4).

\section{DISCUSSION}

The remanent magnetic properties of these basaltic rocks are very unstable, characterized by median

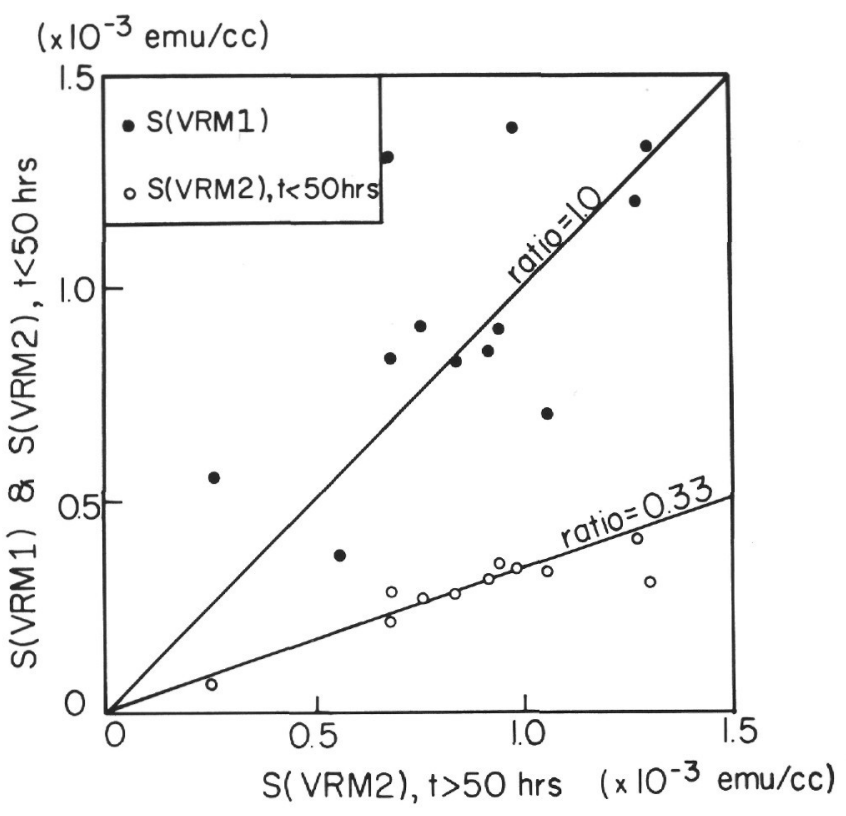

Figure 4. Comparison of magnetic viscosity coefficient magnitudes for VRM1 (S/VRM1]) and the initial stage of VRM2 (S[VRM2], t<50 hr) to the later stage of VRM2 (S[VRM2], $\mathrm{t}>50 \mathrm{hr}$ ) for specimens from Hole $319 \mathrm{~A}$ and Site 321. Lines of constant ratio are shown for reference.

destructive fields less than 100 oe in every sample. The NRM are unstable in direction as well as in intensity. DSDP basalts with these unstable remanent characteristics have been found in various areas of the world ocean and are known to be very susceptible to acquisition of VRM, to the extent that the NRM intensity can be often attributed largely to VRM (Lowrie, 1973, 1974; Lowrie et al., 1973).

The intensity of VRM acquired in $500 \mathrm{hr}$ in the specimens after AF demagnetization was only about half the VRM intensity acquired in the presence of the NRM (Figure 5, Table 2). The previous reports of VRM in DSDP basalts (Lowrie, 1973, 1974) were based on VRM acquired from the demagnetized state. The present results suggest that DSDP basalt of these earlier studies might have shown even more viscous behavior if VRM studies had been carried out prior to AF demagnetization.

Whether acquired from the demagnetized or NRM state, the 500-hr VRM amounted to a very large fraction of the NRM intensity (Table 2). This was especially true in Hole 319A where, prior to demagnetization, the VRM in a 1-oe field was much greater than the NRM in almost every specimen. With the exception of Sample $319 \mathrm{~A}-3-4,136 \mathrm{~cm}$, the time at which the acquired VRM1 equaled the original NRM varied from only 9 to $87 \mathrm{hr}$; that is, from a few hours to a few days.

Ade-Hall (this volume) has postulated from preliminary analysis of remanent magnetic properties that the Hole 319A basalt samples possessed a large vertical component of magnetization which may be induced by the drilling process. It is possible that this vertical component of magnetization is a stress-induced effect pro- 


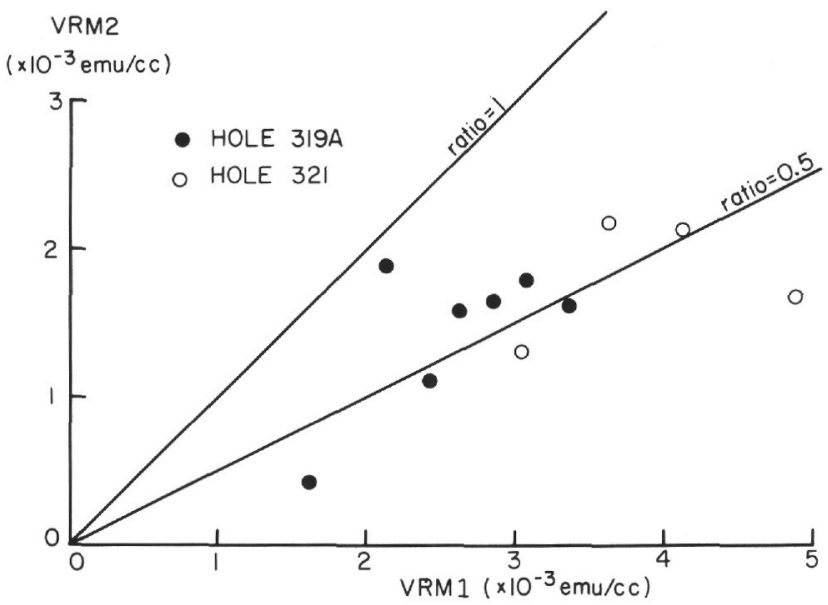

Figure 5. Intensity of VRM acquired in a 1.0-oe constant field over $500 \mathrm{hr}$ before (BRM1) and after (VRM2) $A F$ demagnetization of NRM in specimens from Hole $319 \mathrm{~A}$ and Site 321. Lines of constant ratio are shown for reference.

duced by the drilling process. It is also possible, however, that the vertical component is a VRM acquired while drilling the hole.

The ambient field inside the drill pipe is presumably axial and has an intensity of a few oersteds. VRM would be acquired much more rapidly in the drill pipe remanent field than in the 1-oe laboratory field. This is because VRM $(t)=s \log t$, where $s$ (the magnetic viscosity coefficient) is linearly proportional to field. Thus, a VRM acquired in a 1-oe field in $100 \mathrm{hr}$ (as in the laboratory experiments) would be acquired in a 4-oe field in $(100)^{1 / 4} \mathrm{hr}$, that is, in only slightly more than $3 \mathrm{hr}$. The NRM intensities at Hole 319A (with one exception) could be attributed to VRM acquired in a 1-oe field in 9$90 \mathrm{hr}$ (Table 2). Clearly, in a field of only a few oersteds a VRM equivalent to the NRM could be acquired in a time comparable to that required to drill the hole.

This is only one possible interpretation of the Hole 319A vertical component of magnetization. Further work is needed to test the effects of stresses caused by drilling on the remanent magnetic properties of unstable oceanic basalts. Also, this is the only reported instance where a consistent vertical component of magnetization has been found in DSDP basalts. It is also one of the most unstable oceanic basalts yet studied and showed the most strongly viscous character. Unstable magnetic properties are common in certain oceanic basalts (Lowrie, 1974), although they do not appear to be important in basalts that characterize regions with well-defined linear magnetic anomaly patterns.

\section{ACKNOWLEDGMENTS}

We wish to thank R.L. Larson and N.D. Opdyke for critical reading of the manuscript. Maria Panisello and Doris Lafferty made the remanence and Curie temperature measurements on these rocks. This research was supported under a grant from the Office of Naval Research, Contract N-00014-67-A-01080004 .

\section{REFERENCES}

Ade-Hall, J.M., Palmer, H.C., and Hubbard, T.P., 1971. The magnetic and opaque petrologic response of basalts to regional hydrothermal alteration: Geophys. J., v. 24, p. 137-174.

Creer, K.M., Ibbetson, J., and Drew, W., 1970. Activation energy of cation migration in titanomagnetites: Geophys. J., v. 19, p. 93-101.

Larson, R.L. and Lowrie, W., in press. Paleomagnetic evidence for motion of the Pacific plate from Leg 32 basalts and magnetic anomalies. In Larson, R.L., Moberly, R., et al., Initial Reports of the Deep Sea Drilling Project, Volume 32: Washington (U.S. Government Printing Office).

Lowrie, W., 1973, Viscous remanent magnetization in ocean basalts: Nature, v. 234, p. 27-30.

Lowrie, W., 1974. Oceanic basalt magnetic properties and the Vine and Matthews hypothesis: Z. Geophys., v. 40, p. 513536.

Lowrie, W. and Hayes, D.E., 1975. Magnetic properties of oceanic basalt samples. In Hayes, D., Frakes, L.A., et al., Initial Reports of the Deep Sea Drilling Project, Volume 28: Washington (U.S. Government Printing Office), p. 869-878.

TABLE 2

\begin{tabular}{|c|c|c|c|c|c|c|}
\hline \multirow{2}{*}{$\begin{array}{c}\text { Sample } \\
\text { (Interval in } \mathrm{cm} \text { ) }\end{array}$} & \multicolumn{3}{|c|}{$\begin{array}{l}\text { Magnetization Intensity } \\
\qquad\left(10^{-3} \mathrm{G}\right)\end{array}$} & \multirow{2}{*}{$\begin{array}{l}\text { VRM1 } \\
\text { NRM }\end{array}$} & \multirow{2}{*}{$\begin{array}{l}\text { VRM2 } \\
\text { NRM }\end{array}$} & \multirow{2}{*}{$\begin{array}{c}\text { Time at } \\
\text { which } \\
\text { VRM1 = } \\
\text { NRM (hr) }\end{array}$} \\
\hline & NRM & VRM1 & VRM2 & & & \\
\hline \multicolumn{7}{|l|}{ Hole 319A } \\
\hline $\begin{array}{l}3-1,89 \\
3-2,9 \\
3-2,70 \\
3-3,64 \\
3-4,37 \\
3-4,136 \\
3-5,72\end{array}$ & $\begin{array}{l}2.03 \\
1.71 \\
1.10 \\
1.58 \\
1.83 \\
2.57 \\
1.25\end{array}$ & $\begin{array}{l}2.85 \\
3.07 \\
1.62 \\
2.43 \\
3.36 \\
2.61 \\
2.12\end{array}$ & $\begin{array}{l}1.65 \\
1.79 \\
0.41 \\
1.11 \\
1.62 \\
1.58 \\
1.87\end{array}$ & $\begin{array}{l}1.40 \\
1.80 \\
1.47 \\
1.54 \\
1.84 \\
1.02 \\
1.70\end{array}$ & $\begin{array}{l}0.81 \\
1.05 \\
0.37 \\
0.70 \\
0.89 \\
0.61 \\
1.50\end{array}$ & $\begin{array}{r}87 \\
15 \\
56 \\
44 \\
9 \\
490 \\
37\end{array}$ \\
\hline \multicolumn{7}{|l|}{ Site 321} \\
\hline $\begin{array}{l}14-1,55 \\
14-2,50 \\
14-3,1 \\
14-4,12\end{array}$ & $\begin{array}{r}4.78 \\
10.20 \\
5.64 \\
7.79\end{array}$ & $\begin{array}{l}3.04 \\
4.89 \\
4.13 \\
3.64\end{array}$ & $\begin{array}{l}1.31 \\
1.67 \\
2.13 \\
2.18\end{array}$ & $\begin{array}{l}0.64 \\
0.48 \\
0.73 \\
0.47\end{array}$ & $\begin{array}{l}0.27 \\
0.16 \\
0.38 \\
0.28\end{array}$ & $\begin{array}{l}- \\
- \\
- \\
-\end{array}$ \\
\hline
\end{tabular}


Lowrie, W., Lovlie, R., and Opdyke, N.D., 1973. Magnetic properties of Deep Sea Drilling Project basalts from the North Pacific Ocean: J. Geophys. Res., v. 78, p. 7647-7660.

Ozima, M. and Ozima, M., 1971. Characteristic thermomagnetic curve in submarine basalts: J. Geophys. Res., v. 76, p. 2051-2056.
1972. Activation energy of unmixing of titanomaghemite: Phys. Earth Planet. Interiors, v. 5, p. 87-89.

Wilson, R.L. and Watkins, N.D., 1967. Correlation of petrology and natural magnetic polarity in Columbia Plateau basalts: Geophys. J., v. 12, p. 405-424. 